\title{
To die or not to die:
}

Calpain drives Alzheimer's Disease and cancer progression

\author{
Stacy Visser-Grieve
}

\section{Queen's University}

It's inevitable: we will all age; we will all grow old. And we all know the characteristic signs of aging: wrinkly skin, loss of hearing, loss of memory, and increased risk of acquiring certain diseases. Two of the diseases commonly associated with aging are Alzheimer's disease (AD) and cancer. Interestingly, studies show an inverse relationship between the development of cancer and $A D$, such that patients suffering from dementia often have a decreased occurrence of cancer. ${ }^{1}$ Although at first this may seem surprising, in fact, the mechanisms mediating progression of these diseases are inherently connected. In particular, each disease originates from a defect in programmed cell death, or apoptosis. Whereas $A D$ results in neurodegeneration from enhanced cell death, cancer is often associated with pro-survival or anti-apoptotic effects. Importantly, the protease calpain plays a pivotal role in modulating both pro- and anti-apoptotic functions depending on cellular context ${ }^{2}$ and thus may act as a driver in the development of Alzheimer's disease and cancer.

Calpain belongs to a family of intracellular $\mathrm{Ca}^{2+}$-activated cysteine proteases - proteins that proteolyze or cleave their substrate proteins. Of the 14 mammalian isoforms, calpain 1 and calpain 2 (herein referred to collectively as calpain) are ubiquitously expressed and the most extensively studied. ${ }^{3}$ By cleaving its numerous substrates into smaller functioning fragments, calpain modulates many cellular phenotypes including cell cycle progression, adhesion, migration, and survival. Due to its essential role in maintaining cellular homeostasis, the activity of calpain is tightly regulated. Primarily, $\mathrm{Ca}^{2+}$ binding activates calpain by allowing for conformational changes that enhance cleavage of available substrates. Alternatively, binding of the endogenous inhibitor calpastatin prevents proteolytic activity. $^{3}$

Several studies show that calpain expression is upregulated in specific cancers, including colorectal, breast, or prostate cancers, and it may correlate with poor progression. ${ }^{4}$ These studies suggest that calpain primarily plays a pro-tumorigenic role. Similarly, calpain also promotes Alzheimer's disease. By examining the brains of AD patients, calpain was shown to be hyperactivated due to increased levels of $\mathrm{Ca}^{2+}$ and/or decreased expression of calpain's endogenous inhibitor calpastatin. ${ }^{5}$

The ability of calpain to mediate either pro- or antiapoptotic functions may explain how increased calpain activity can promote $A D$ and cancer. Firstly, in $A D$, increased calpain activity occurs early and therefore contributes to multiple aspects of neurodegeneration. In addition to contributing to the formation of clinical hallmarks such as senile plaques containing beta amyloid $(A \beta)$, and neurofibrillary tangles composed of hyperphosphorylated microtubule associated protein tau - calpain also directly activates cell death pathways in neurons. ${ }^{2,5}$ Specifically, calpain decreases activity of anti-apoptotic proteins such as $\mathrm{Bcl}-2$ or activates pro-apoptotic proteins including p53 and Bax. ${ }^{6}$ Increased calpain activity enhances cleavage of amyloid precursor protein into $A \beta$, contributing to the formation of $A \beta$ plaques. Additionally, calpain specifically cleaves kinases such as GSK- $\beta$ and CDK5, enhancing their ability to phosphorylate tau, leading to neurofibrillary tangle formation. Finally, calpain specifically cleaves tau into smaller fragments that signal the induction of apoptosis. ${ }^{5,6}$ These are some examples of how increased calpain activity in the brain can lead to neurodegeneration. These changes in neuropathology potentiated by calpain inevitably lead to the loss of neurons, shrinkage and loss of neuronal processes, and dramatic loss of brain volume observed in AD patients.

In contrast with $A D$, tumorigenesis involves the overgrowth of cells resulting from increased cell cycle progression and/or loss of programmed cell death. In this disease setting, evidence is emerging that calpain promotes cell $\downarrow$ 
c survival. For example, in breast cancer cells, loss of calpain 2 expression leads to reduced activity of the pro-survival kinase AKT. ${ }^{7}$ Furthermore, loss of both calpain 1 and 2 increased starvation-induced cell death, and it also reduced activity of the AKT survival pathway. ${ }^{8}$ Similarly, increased expression of calpain has been associated with resistance to certain drugs, including the targeted therapeutic Herceptin in breast cancer. ${ }^{9}$ These results provide a novel hypothesis that calpain may play a pro-survival function in cancer cells as opposed to an anti-apoptotic role in normal cells.

If this hypothesis holds true, calpain could be an important therapeutic target for both $A D$ and cancer. Using an $A D$ mouse model, Medeiros et al recently showed that treating transgenic $A D$ mice with a calpain inhibitor could reduce accumulation of $A \beta$ plaques, decrease tau phosphorylation, and improve cognitive function. ${ }^{10}$ Similar studies using calpain inhibitors in cancer settings showed how inhibiting calpain enhanced cell death in cancer cells ${ }^{11}$ or downregulating calpain 2 expression reduced tumor growth in mouse models. ${ }^{7}$

Calpain drives progression of either Alzheimer's disease or cancer through its pro- and anti-apoptotic effects, respectively. Understanding the cellular context and the substrates through which calpain acts to elicit these seemingly opposing roles are current research areas garnering interest by scientists in both the $A D$ and cancer fields. If calpain can manipulate its functions depending upon cellular context and available substrates, the question follows: are other diseases also driven by calpain?

\section{References}

1. Behrens $\mathrm{Ml}$, Lendon $\mathrm{C}$, Roe CM. A common biological mechanism in cancer and Alzheimer's disease? Curr Alzheimer Res. 2009;6(3):196-204.

2. Tan Y, Wu C, De Veyra T, Greer PA. Ubiquitous calpains promote both apoptosis and survival signals in response to different cell death stimuli. J. Biol. Chem. 2006;281(26):17689-98.

3. Goll DE, Thomspon VF, Li H, Wei W, Cong J. The calpain system. Physiol Rev. 2003;83(3):731-801

4. Storr, SJ, Carragher NO, Frame MC, Parr T, Martin SG. The calpain system and cancer. Nat. Reviews. Cancer. 2011;11(5):364-74.

5. Ferreira A. Calpain dysregulation in Alzheimer's disease. ISRN Biochemistry. 2012. doi: 10:5402/2012/728571

6. Raynaud F, Marcilhac A. Implication of calpain in neuronal apoptosis: A possible regulation of Alzheimer's disease. FEBS J. 2006;273(15);3437-43.

7. Ho WC, Pikor L, Gao Y, Elliott BE, Greer PA. Calpain 2 regulates Akt-FoxOp27(Kip1) protein signaling in mammary carcinoma. J. Biol. Chem. 2012;287 (19):15458-65.

8. Bertoli C, Copetti T, Lam EWF, Demarchi F, Schneider C. Calpain small-1 modulates Akt/FoxO3A signaling and apoptosis through PP2A. Oncogene. 2009;28(5):721-33.

9. Kulkarni S, Reddy KB, Esteva FJ, Moore HC, Budd GT, Tubbs RR. Calpain regulates sensitivity to trastuzumab and survival in HER2-positive breast cancer. Oncogene 2012;29(9):1339-50.

10. Medeiros R, Kitazawa M, Chabrier MA, Cheng D, Baglietto-Vargas D, Kling A, et al. Calpain inhibitor A-705253 mitigates Alzheimer's disease-like pathology and cognitive decline in aged 3xTgAD mice. Am J Pathol. 2012; 181(2):616-25.

11. Guan NA Korukonda R, Hurh E, Schmittgen TD, Donkor IO, Dalton JT. Apoptosis induced by novel aldehyde calpain inhibitors in human tumor cell lines. Int J Oncol. 2006;29(3):633-63.

\section{Stacy Visser-Grieve}

Stacy Visser-Grieve completed her PhD in the Department of Pathology and Molecular Research at Queen's University studying the role of the Hippo signaling pathway in tumorigenesis. She is currently funded by the Canadian Breast Cancer Foundation-Ontario Chapter for her post-doctoral work on the therapeutic implications of protease calpain in breast cacner at Queen's Cancer Research Institute. Stacy is a life-long learner and enjoys delving into the literature to make connections between various signaling networks within different disease settings. 